\title{
MONOTONE $n$-FRAMES ARE TAME ${ }^{1}$
}

\section{RICHARD GILLETTE AND JAMES VAN BUSKIRK}

An $n$-frame $\Im_{n}$ is the union of $n$ arcs which are disjoint except for a common end point called the branch point of $\mathcal{F}_{n}$. An $n$-frame of $E^{3}$ is tamely imbedded provided there is an autohomeomorphism of $E^{3}$ which carries it onto a polygonal $n$-frame. We will say that an $n$-frame $\mathcal{F}_{n}$ in $E^{3}$ is monotone provided each geometric 2 -sphere centered at the branch point of $\mathcal{F}_{n}$ intersects each of the $n$ defining arcs of $\mathcal{F}_{n}$ in at most one point. Because each monotone $n$-frame is of the same imbedding type as a monotone $n$-frame having its branch point at the origin and its end points on the unit sphere, we will assume that a monotone $n$-frame has these properties as well as a prescribed ordering, say $a_{1}, a_{2}, \cdots, a_{n}$, of its defining arcs.

Since for each $s \in(0,1]$, the arc $a_{i}$ intersects the sphere of radius $s$ centered at the origin in a single point $p_{i}(s)$, the equation $\phi(s)$ $=\left(p_{1}(s), \cdots, p_{n}(s)\right), 0<s \leqq 1$, defines a continuous function from $(0,1]$ into the space $F_{n}\left(S^{2}\right)$ of all $n$-tuples of distinct points of $S^{2}$. In this way there is established a one-one correspondence between monotone $n$-frames $F_{n}$ and continuous maps $\phi$ of $(0,1]$ into $F_{n}\left(S^{2}\right)$. We restrict our attention to frames $\mathcal{F}_{n}$ for which the $n$-tuple of end points $\left(p_{1}(1), \cdots, p_{n}(1)\right)$ is the base point $\left(p_{1}, \cdots, p_{n}\right)$ of $F_{n}\left(S^{2}\right)$.

Let $L H\left(B^{3}\right)$ be the space of all orientation preserving autohomeomorphisms $H$ of the unit ball $B^{3}$ which are level preserving in the sense that $\|H(x)\|=\|x\|$ for all $x \in B^{3}$. To each $H \in L H\left(B^{3}\right)$ there corresponds a family $h_{s}$ of autohomeomorphisms of the boundary sphere $S^{2}$ of $B^{3}$ defined by $h_{s}(x)=H(s x) / s$ for $s \in(0,1], x \in S^{2}$. Therefore $L H\left(B^{3}\right)$ is in one-one correspondence with the set of maps of $(0,1]$ into the group $G\left(S^{2}\right)$ of all orientation preserving autohomeomorphisms of $S^{2}$.

In order to relate monotone $n$-frames $\mathcal{F}_{n}$ and level preserving homeomorphisms $H$, consider the map $\rho_{n}: G\left(S^{2}\right) \rightarrow F_{n}\left(S^{2}\right)$ defined by $\rho_{n}(f)=\left(f\left(p_{1}\right), \cdots, f\left(p_{n}\right)\right)$, where $\left(p_{1}, \cdots, p_{n}\right)$ is the base point of $F_{n}\left(S^{2}\right)$.

The following theorem is patterned after a result of G. S. McCarty [3, Lemma 4.1], but, as used here, is better viewed as a generalization of a fundamental theorem of E. Artin in braid theory [1, p. 104]. (See

Presented to the Society, August 28, 1968; received by the editors July 3, 1967.

${ }_{1}^{1}$ Prepared with partial support from the Office of Scientific and Scholarly Research of the Graduate School of the University of Oregon. 
D. M. Dahm [2, p. 15], where this generalization is developed for braids on arbitrary manifolds.) Although the theorem is stated for $S^{2}$, the proof given is valid for any connected manifold of dimension at least 2 .

THEOREM 1. $G\left(S^{2}\right)$ is a fiber bundle over $F_{n}\left(S^{2}\right)$ relative to the map $\rho_{n}$.

Proof. $G\left(S^{2}\right)$ is an effective transitive group of transformations of $F_{n}\left(S^{2}\right)$. Consequently, by the bundle structure theorem, it suffices to show that $\rho_{n}$ admits a local cross-section. But, since the basic open sets in $F_{n}\left(S^{2}\right)$ may be chosen to be products of $n$ disjoint open discs, this is a consequence of the following elementary fact. Let $G\left(B^{2}, S^{1}\right)$ be the group of autohomeomorphisms of the 2-cell $B^{2}$ which are pointwise fixed on the boundary $S^{1}$, and let $\pi$ be the map of $G\left(B^{2}, S^{1}\right)$ onto the interior of $B^{2}$ defined by $\pi(f)=f(0)$. Then $\pi$ admits a global cross section.

As a corollary to Theorem 1, we have our main result.

THEOREM 2. Any monotone $n$-frame is tame.

Proof. Let $\mathcal{F}_{n}$ be a monotone $n$-frame. Then $\mathscr{F}_{n}$ corresponds to a map $\phi:(0,1] \rightarrow F_{n}\left(S^{2}\right)$. Choosing the base point $\left(p_{1}, \cdots, p_{n}\right)$ to be $\phi(1)$, the map $\phi$ lifts to a map $h:(0,1] \rightarrow G\left(S^{2}\right)$ such that $h_{1}$ is the identity. The corresponding map $H$ in $L H\left(B^{3}\right)$ is an autohomeomorphism of $B^{3}$ carrying $F_{n}$ onto $n$ radial segments in $B^{3}$. Since $H$ is the identity on the boundary sphere, $H$ extends to all of $E^{3}$ and hence $\mathfrak{F}_{n}$ is tame.

AdDED In PROOF. The authors are grateful to Professor R. H. Bing for pointing out that Theorem 2 (generalized, as noted, to $n$-manifolds) is equivalent to Proposition 2.3 of: R. H. Bing and V. L. Klee, Every simple closed curve in $E^{3}$ is unknotted in $E^{4}, \mathrm{~J}$. London Math. Soc. 39 (1964), 86-94.

\section{BIBLIOGRAPHY}

1. E. Artin, Theory of braids, Ann. of Math. 48 (1947), 101-126.

2. D. M. Dahm, A generalization of braid theory, Ph.D. Thesis, Princeton Univ., Princeton, N. J., 1962. 304.

3. G. S. McCarty, Homeotopy groups, Trans. Amer. Math. Soc. 106 (1963), 293-

UNIVERSITY OF OREGON 